Relations industrielles

Industrial Relations

\title{
Eme, B. et J.L. Laville, sous la direction de, Cohésion sociale et emploi
}

\section{Jean Saglio}

Volume 50, numéro 1, 1995

URI : https://id.erudit.org/iderudit/051003ar

DOI : https://doi.org/10.7202/051003ar

Aller au sommaire du numéro

Éditeur(s)

Département des relations industrielles de l'Université Laval

ISSN

0034-379X (imprimé)

1703-8138 (numérique)

Découvrir la revue

Citer ce compte rendu

Saglio, J. (1995). Compte rendu de [Eme, B. et J.L. Laville, sous la direction de, Cohésion sociale et emploi]. Relations industrielles / Industrial Relations, 50(1), 234-235. https://doi.org/10.7202/051003ar

Tous droits réservés @ C Département des relations industrielles de l'Universite Laval, 1995
Ce document est protégé par la loi sur le droit d'auteur. L’utilisation des services d'Érudit (y compris la reproduction) est assujettie à sa politique d'utilisation que vous pouvez consulter en ligne.

https://apropos.erudit.org/fr/usagers/politique-dutilisation/ 


\section{Cohésion sociale et emploi}

sous la direction de B. EME et J.L. LAViLLE, Paris, Ed. Desclée de Brouwer, 1994, 284 p., ISBN 2-220-03524-7.

On connaît bien l'apparent paradoxe de la situation française depuis près de vingt ans maintenant : alors que la croissance économique perdure, même si c'est à un rythme plus faible que dans l'après Seconde Guerre mondiale, la situation de l'emploi ne cesse de se dégrader et l'exclusion sociale progresse. Du fait de cette déconnexion entre progrès économique et cohésion sociale, bien des observateurs en déduisent que, plus qu'une simple crise économique, c'est donc bien le mode de régulation de la société qui est en cause. Ce qui signifie aussi, comme le note Renaud Sainsaulieu dans la préface de cet ouvrage, que les mécanismes identitaires traditionnels, c'est à dire en fait ceux que la société française de l'industrialisation avait fini par adopter, ne sont plus pertinents. Face à de telles ruptures identitaires, c'est une imagination sociale forte qui est requise : il faut donc réinventer les mécanismes de la cohésion sociale, et les articuler à de nouvelles définitions de l'emploi et du travail. Contribuer à cette construction des nouvelles solidarités, tel est bien l'objectif, ambitieux et généreux de cet ouvrage collectif.

Trois grands thèmes structurent l'ouvrage. Le premier se veut analytique: la crise que nous traversons est en effet multiforme et il s'agit de prendre la mesure de ses effets dans leurs différentes dimensions et dans toute leur ampleur. Ainsi, pour progresser, explique Guy Roustang, il nous faut procéder à une mise en cause des modes de penser et des catégories devenus quasi naturels auxquels l'éonomie classique nous a habitués. Mais déjà on peut observer que certaines de ces catégories qui paraissaient immuables ont été bouleversées. C'est ce que montre Xavier Gaullier quand il examine les changements considérables qui affectent les modes de gestion du travail, de la carrière, les articulations entre travail et hors travail, les cycles de vie. Pour Michel Lallement ce sont encore les remèdes classiques des politiques économiques qu'il convient de repenser: l'État français traverse manifestement une grave "panne sociale" en matière de politique de l'emploi.

Parmi les préconisations les plus habituelles, le "partage du travail " occupe une place éminente liée à la simplicité des arithmétiques sociales sur lesquelles il se fonde : puisque l'emploi se fait rare, pourquoi pas le partager? Voire, puisque la quantité de richesses produites ne cesse de progresser, pourquoi ne pas dissocier travail et revenu de base par un mécanisme d'allocation universelle? Daniel Mothé produit une critique vigoureuse et argumentée de ces "utopies du temps libre " en soulignant combien ces gentils contes de fées oublient le plus souvent la nature politique et sociale, faite de conflits, de contraintes, de pouvoirs et de violences, de la cohésion sociale dont ils déplorent la disparition.

Le thème de la seconde partie de l'ouvrage est plus prospectif : que pourrait donc être une économie solidaire? Jean Louis Laville préconise de rompre les cercles vicieux de la marchandisation en privilégiant le rôle des acteurs sur celui des marchés et en organisant l'intégration de l'" économique " et du "social" et non leur cloisonnement. Bernard Eme, à partir d'une analyse critique des politiques d'insertion, tente d'expliciter comment la cohésion sociale peut et doit se reconstruire à un niveau local, autour des projets et de pratiques reposant pour beaucoup sur la confiance. À côté de ces deux textes plutôt programmatiques, Jean François Colin nous propose l'analyse d'une expérience concrète: celle des cafés 
musiques implantés dans des quartiers en difficulté et Jean Baptiste de Foucauld, en quelques pages sommaires, rappelle les principaux enjeux de tous ces projets.

La troisième partie se veut plus politique. $\grave{A}$ partir d'une analyse classique des problèmes de la reconnaissance institutionnelle par les pouvoirs publics dans la situation allemande actuelle, Adalbert Evers pose en conclusion la question tout à fait pertinente du divorce grandissant entre les idéologies politiques libérales et la nécessité de reconnaître un rôle clef aux institutions de solidarité. À partir de quelques expériences québécoises et avec beaucoup de pragmatisme, Benoit Lévesque dégage quelques formes de reconnaissance institutionnelle effectivement mises en œuvre outre-Atlantique. Laurent Thévenot, pour sa part, montre l'inadaptation des catégories de pensée administratives et statistiques pour raisonner l'économie solidaire. Enfin, Bernard Perret, se fondant sur l'expérience du Conseil de l'Évaluation, tente d'expliciter l'ampleur des changements culturels nécessaires au niveau de l'État et de la haute fonction publique pour parvenir à cette solidarité.

Comme c'est bien souvent le cas dans de tels ensembles à plusieurs voix, on peut facilement gloser sur les différences de points de vue entre les auteurs. Les définitions avancées de l'économie solidaire et de la cohésion sociale sont effectivement diverses et variées. Probablement ne sont-elles pas véritablement antinomiques, mais certainement pas non plus totalement cohérentes. C'est bien ainsi que progresse le débat public.

Plus surprenant, par contre, est le fait que l'ensemble de ces auteurs semblent partager, au moins implicitement, l'idée que les catégories de penser les liens entre l'" économique " et le "social " telles que définies par l'économie classique auraient été admises communément. Après tout, il existe bien tout un courant de pensée, allant des philosophes et des anthropologues aux économistes, qui considère bien que les catégories de la pensée, tout autant que celles de la pratique sont des produits sociaux et que même les structures économiques les plus fondamentales, tout comme les modèles économiques les plus abstraits, sont des produits historiques et contingents. Le choix de considérer l'économie comme la science des rapports des hommes aux choses ("à la nature") n'est spécifique qu'à une des branches de la théorie économique. Qu'elle soit aujourd'hui la branche dominante ne doit pas pour autant faire oublier qu'il en existe d'autres et que l'établissement même de cette domination fut un long travail social. $\grave{A}$ ainsi reconnaître les apports de tels précurseurs, et à tenter de comprendre pourquoi on les aurait oubliés, on pourrait peut-être se donner les moyens de penser réellement les raisons sociales pour lesquelles la société française considère les problèmes sociaux actuels comme inéluctables.

JeAN SAGLIO CNRS, Université Lyon II

\section{Occupational Subcultures in the Workplace}

par Harrison M. Trice, Ithaca, New York, ILR Press, 1993, 287 p., ISBN 087546-302-9 (cloth) and ISBN 0-87546-303-7.

$\mathrm{Au}$ cours des deux dernières décennies, nous avons assisté au développement fulgurant d'une littérature sur la réalité culturelle des organisations. Règle générale, ces publications, tant d'ordre conceptuel que d'ordre empirique, ren- voient à une vision globale, monolithique de la culture organisationnelle, faisant peu ou pas de place à l'expression d'idéologies, de valeurs ou de croyances qui sont partagées par des groupements restreints au sein même des 